\title{
Comment on the work of Zhang et al. (2017, Journal of Inequalities and Applications)
}

\section{Yiqing Chen ${ }^{1 *}$ (D)}

\section{${ }^{*}$ Correspondence: \\ yiqing.chen@drake.edu \\ ${ }^{1}$ College of Business and Public Administration, Drake University, Des Moines, USA}

\begin{abstract}
In a recent work by Zhang et al. (J. Inequal. Appl. 2017:82, 2017) the authors studied large deviations of the aggregate amount of claims in a size-dependent renewal risk model. They claimed that they obtained a precise large-deviation formula for the case with subexponential claims and with arbitrary dependence between each claim size and its waiting time. In this short note, we point out that their main result is unfortunately not correct in general.
\end{abstract}

MSC: Primary 60F10; secondary 91B30; 60K05

Keywords: Aggregate amount of claims; Precise large deviations; Subexponential class; Dependence; Renewal counting process; Counterexample

\section{Review of the work by Zhang et al. [7]}

Large deviations of the aggregate amount of claims are an important topic, which was initiated by Klüppelberg and Mikosch [4], reactivated by Tang et al. [6], and revisited by many researchers afterward. Due to its potential applications to insurance and finance, this topic is receiving increasing attention from academia.

In a recent work by Zhang et al. [7] the authors studied large deviations of the aggregate amount of claims in a size-dependent renewal risk model. In their notation, let $\left\{X_{k}, k \in \mathbb{N}\right\}$ and $\left\{\theta_{k}, k \in \mathbb{N}\right\}$ be claim sizes and interarrival times, respectively. Assume that the pairs $\left(X_{k}, \theta_{k}\right), k \in \mathbb{N}$, form a sequence of independent and identically distributed (i.i.d.) copies of a generic random pair $(X, \theta)$ with marginal distribution functions $F=1-\bar{F}$ on $[0, \infty)$ and $G$ on $[0, \infty)$ and with arbitrary dependence between $X$ and $\theta$. Define an integer-valued stochastic process

$$
N_{t}^{*}=\inf \left\{k \in \mathbb{N}: \theta_{1}+\cdots+\theta_{k} \geq t\right\}, \quad t \geq 0 .
$$

Note that $N_{t}^{*}$ is slightly different from the commonly used renewal counting process

$$
N_{t}=\sup \left\{k \in \mathbb{N}: \theta_{1}+\cdots+\theta_{k} \leq t\right\} .
$$

(c) The Author(s) 2019. This article is distributed under the terms of the Creative Commons Attribution 4.0 International License (http://creativecommons.org/licenses/by/4.0/), which permits unrestricted use, distribution, and reproduction in any medium, provided you give appropriate credit to the original author(s) and the source, provide a link to the Creative Commons license, and indicate if changes were made. 
Then the aggregate amount of claims is defined by

$$
S_{t}^{*}=\sum_{k=1}^{N_{t}^{*}} X_{k}, \quad t \geq 0,
$$

where the sum is understood as 0 when $N_{t}^{*}=0$.

The authors consider the case of subexponential claims. By definition a distribution function $F$ on $[0, \infty)$ is subexponential, denoted by $F \in \mathcal{S}$, if

$$
\lim _{x \rightarrow \infty} \frac{\overline{F^{* n}}(x)}{\bar{F}(x)}=n
$$

for all $n \geq 2$, where $F^{* n}$ denotes the $n$-fold convolution of $F$. As the authors pointed out, (1.1) implies

$$
\lim _{x \rightarrow \infty} \frac{P\left(X_{1}+\cdots+X_{n}>x\right)}{P\left(\max \left\{X_{1}, \ldots, X_{n}\right\}>x\right)}=1
$$

where $X_{1}, X_{2}, \ldots$ are i.i.d. random variables with common distribution function $F$.

Then the authors stated the following precise large-deviation result.

Theorem ZWY Assume that $F \in \mathcal{S}, E[X]=\mu \in(0, \infty)$, and $E[\theta]=1 / \lambda \in(0, \infty)$. Then for arbitrarily given $\gamma>0$, it holds uniformly for all $x \geq \gamma$ t that

$$
P\left(S_{t}^{*}-\mu \lambda t>x\right) \sim \lambda t \bar{F}(x), \quad t \rightarrow \infty .
$$

Here the uniformity is understood as

$$
\lim _{t \rightarrow \infty} \sup _{x \geq \gamma t}\left|\frac{P\left(S_{t}^{*}-\mu \lambda t>x\right)}{\lambda t \bar{F}(x)}-1\right|=0 .
$$

This result is claimed to hold for the whole subexponential class $\mathcal{S}$, and, in particular, it squarely removes a condition on the dependence structure of $(X, \theta)$ originally proposed by Chen and Yuen [2] and recently used by many researchers. Thus this result, if correct, would be an important contribution to the theory of large deviations. Unfortunately, the counterexample given in the following section disproves it.

\section{A counterexample}

Assume that:

(i) the generic random pair $(X, \theta)$ contains comonotonic and identical components, that is, $X=\theta$;

(ii) the common distribution of $X$ and $\theta$ is the Weibull distribution

$$
F(x)=1-e^{-\sqrt{x}}, \quad x \geq 0
$$

(iii) $x=t \rightarrow \infty$. 
Then

$$
P\left(S_{t}^{*}-\mu \lambda t>x\right) \sim \lambda \int_{x}^{2 x} \bar{F}(y) d y=o(\lambda t \bar{F}(x))
$$

Our proof given in the next section shows that we have plenty of room to generalize this counterexample, but we will not do so to save space. Thus Theorem ZWY is fatally wrong. The erroneous step appears on the first two lines of their proof, where the authors claimed that, by the assumption $F \in \mathcal{S}$ and relation (1.2), to prove (1.3), one needs only to prove

$$
P\left(\max _{k \leq N_{t}^{*}}\left(X_{k}-\mu\right)>x\right) \sim \lambda t \bar{F}(x), \quad t, x \rightarrow \infty .
$$

They seem to have overlooked an essential difference between (1.2) and (2.2): The index $n$ in (1.2) is arbitrarily fixed, whereas the index $N_{t}^{*}$ in (2.2) varies in $t$ and almost surely diverges to $\infty$ as $t \rightarrow \infty$.

Nevertheless, in the paper the authors developed a martingale approach to the study of precise large deviations, which is novel to us.

\section{Proof of (2.1)}

By conditions (i) and (ii), $\mu=1 / \lambda=12$. By condition (ii), $F$ has a long and rapidly varying tail; see Embrechts et al. [3] for these and related terminologies. Furthermore, for a distribution $F$ with a long and rapidly varying tail, it is easy to verify the following:

$$
\bar{F}(x)=o\left(\int_{x}^{\infty} \bar{F}(y) d y\right), \quad \int_{x}^{2 x} \bar{F}(y) d y \sim \int_{x}^{\infty} \bar{F}(y) d y=o(x \bar{F}(x)) .
$$

See Su and Tang [5] for closely related discussions; for example, the first relation in (3.1) can be found in their Theorem 3.1(i). Thus, it remains to prove the first step in (2.1). Keeping in mind condition (iii), we derive

$$
\begin{aligned}
P\left(S_{t}^{*}-\mu \lambda t>x\right) & =\sum_{n=1}^{\infty} P\left(\sum_{k=1}^{n} X_{k}>2 x, N_{t}^{*}=n\right) \\
& =\sum_{n=1}^{\infty} P\left(\sum_{k=1}^{n} X_{k}>2 x, \sum_{k=1}^{n-1} X_{k}<x\right) \\
& =\int_{0}^{x-} \bar{F}(2 x-y) \sum_{n=1}^{\infty} P\left(\sum_{k=1}^{n-1} X_{k} \in d y\right) \\
& =\int_{0}^{x} \bar{F}(2 x-y) d \lambda(y),
\end{aligned}
$$

where $\int_{0}^{x-}$ is understood as $\int_{(0, x)}$, and $\lambda(y)=E\left[N_{y}\right]$ for $y \geq 0$ is the renewal function. The last step can be verified as follows: for $y>0$,

$$
\sum_{n=1}^{\infty} P\left(\sum_{k=1}^{n-1} X_{k} \leq y\right)=\sum_{n=0}^{\infty} P\left(\sum_{k=1}^{n} X_{k} \leq y\right)=1+\sum_{n=1}^{\infty} P\left(N_{y} \geq n\right)=1+\lambda(y) .
$$


Recall Blackwell's renewal theorem:

$$
\lim _{x \rightarrow \infty}(\lambda(x+1)-\lambda(x))=\lambda
$$

see, e.g., page 155 of Asmussen [1]. Thus, for arbitrarily fixed small $\varepsilon>0$, there is some large $x_{0} \in \mathbb{N}$ such that, for all $x \geq x_{0}$,

$$
(1-\varepsilon) \lambda \leq \lambda(x+1)-\lambda(x) \leq(1+\varepsilon) \lambda .
$$

We continue the derivation:

$$
\begin{aligned}
P\left(S_{x}^{*}-\mu \lambda x>x\right) & =\left(\int_{0}^{x_{0}}+\sum_{i=x_{0}}^{\lfloor x\rfloor-1} \int_{i}^{i+1}+\int_{\lfloor x\rfloor}^{x-}\right) \bar{F}(2 x-y) d \lambda(y) \\
& =I_{1}+I_{2}+I_{3},
\end{aligned}
$$

where $\lfloor x\rfloor$ is the commonly used floor function. Since $F$ has a long tail,

$$
I_{1} \sim \bar{F}(2 x) \lambda\left(x_{0}\right)
$$

For the other two terms in (3.3), we derive

$$
\begin{aligned}
I_{2}+I_{3} & \leq \sum_{i=x_{0}}^{\lfloor x\rfloor} \int_{i}^{i+1} \bar{F}(2 x-y) d \lambda(y) \\
& \leq \sum_{i=x_{0}}^{\lfloor x\rfloor} \bar{F}(2 x-i-1)(\lambda(i+1)-\lambda(i)) \\
& \leq(1+\varepsilon) \lambda \sum_{i=x_{0}}^{\lfloor x\rfloor} \bar{F}(2 x-i-1) \\
& \leq(1+\varepsilon) \lambda \sum_{i=x_{0}}^{\lfloor x\rfloor} \int_{i}^{i+1} \bar{F}(2 x-y-1) d y \\
& \leq(1+\varepsilon) \lambda \int_{0}^{x+1} \bar{F}(2 x-y-1) d y \\
& =(1+\varepsilon) \lambda \int_{x-2}^{2 x-1} \bar{F}(y) d y \\
& \sim(1+\varepsilon) \lambda \int_{x}^{2 x} \bar{F}(y) d y,
\end{aligned}
$$

where in step (3.4) we applied the upper bound in (3.2), and in step (3.5) we used the long tail property of $F$ again and the two asymptotic relations in (3.1). Plugging these estimates into (3.3) yields

$$
P\left(S_{x}^{*}-\mu \lambda x>x\right) \lesssim \bar{F}(2 x) \lambda\left(x_{0}\right)+(1+\varepsilon) \lambda \int_{x}^{2 x} \bar{F}(y) d y \sim(1+\varepsilon) \lambda \int_{x}^{2 x} \bar{F}(y) d y,
$$


where the last step is due to $\bar{F}(2 x) \leq \bar{F}(x)=o\left(\int_{x}^{2 x} \bar{F}(y) d y\right)$. A similar asymptotic lower bound can also be established. Finally, by the arbitrariness of $\varepsilon$ we prove the first step in (2.1).

\section{Acknowledgements}

The author wishes to thank the two anonymous referees for their very careful reading of the previous version of the paper.

\section{Funding}

The research was supported by a Center of Actuarial Excellence (CAE) Research Grant (2018-2021) from the Society of Actuaries (SOA) and a Summer Research Grant from the College of Business and Public Administration, Drake University.

Availability of data and materials

Not applicable.

\section{Competing interests}

The author declares that she has no competing interests.

\section{Author's contributions}

This entire work has been completed solely by the author. Author read and approved the final manuscript.

\section{Publisher's Note}

Springer Nature remains neutral with regard to jurisdictional claims in published maps and institutional affiliations.

Received: 9 February 2019 Accepted: 24 June 2019 Published online: 04 July 2019

\section{References}

1. Asmussen, S.: Applied Probability and Queues. Springer, Berlin (2003)

2. Chen, Y., Yuen, K.C.: Precise large deviations of aggregate claims in a size-dependent renewal risk model. Insurance Math. Econom. 51(2), 457-461 (2012)

3. Embrechts, P., Klüppelberg, C., Mikosch, T.: Modelling Extremal Events: For Insurance and Finance. Springer, Berlin (1997)

4. Klüppelberg, C., Mikosch, T.: Large deviations of heavy-tailed random sums with applications in insurance and finance. J. Appl. Probab. 34(2), 293-308 (1997)

5. Su, C., Tang, Q.: Characterizations on heavy-tailed distributions by means of hazard rate. Acta Math. Appl. Sin. 19(1), 135-142 (2003)

6. Tang, Q., Su, C., Jiang, T., Zhang, J.: Large deviations for heavy-tailed random sums in compound renewal model. Statist. Probab. Lett. 52(1), 91-100 (2001)

7. Zhang, S., Wang, D., Yu, S.: Precise large deviations of aggregate claims in a size-dependent renewal risk model with stopping time claim-number process. J. Inequal. Appl. 2017, 82 (2017)

\section{Submit your manuscript to a SpringerOpen ${ }^{\circ}$ journal and benefit from:}

- Convenient online submission

- Rigorous peer review

- Open access: articles freely available online

- High visibility within the field

- Retaining the copyright to your article 